\title{
Characterization of the thermoelastic martensitic transformation in a NiTi alloy driven by temperature variation and external stress
}

\author{
K. F. Liang \\ Department of Physics and Centre of Materials Science, The University of Hong Kong, Pokfulam Road, Hong Kong \\ and Department of Physics, Zhongshan University, Guangzhou 510275, China \\ Z. C. Lin \\ Department of Physics, Zhongshan University, Guangzhou 510275, China \\ P. C. W. Fung \\ Department of Physics and Centre of Materials Science, The University of Hong Kong, Pokfulam Road, Hong Kong \\ J. X. Zhang \\ Department of Physics, Zhongshan University, Guangzhou 510275, China \\ (Received 29 May 1996; revised manuscript received 7 October 1996)
}

\begin{abstract}
In order to test the concept of the physics of dissipation during first-order phase transitions in solids, we measured the internal friction $\left(Q^{-1}\right)$ and the relative shear modulus $(\mu)$ during a thermoelastic martensitic transformation in a NiTi alloy. We adopted two approaches: temperature variation and application of external stress. This investigation of internal friction was carried out with various vibration frequencies $\omega$, temperature variation rates $\dot{T}$, and strain variation rates $\dot{\varepsilon}$. The index $l$ (coupling factor between phase interface and oscillating stress) and index $n$ (rate exponent for the effective phase transformation driving force) have been calculated from the experimental data for each case and the values of $l$ and $n$ are about the same in the two (doped) NiTi samples, irrespective of whether the phase transition is driven by a temperature variation or stress induced process. We compare the values of $n$ and $l$ for the NiTi samples with that of the other samples $\left(\mathrm{VO}_{2}\right.$ ceramics and FeMn alloys), reinforcing the previous physical interpretations of these indices. We believe the indices $n$ and $l$ are indeed fingerprints of first-order phase transitions in solids.
\end{abstract}

[S0163-1829(97)00230-0]

\section{INTRODUCTION}

A theory of dissipation associated with a first-order phase transformation (FOPT) in solids was formulated recently, ${ }^{1}$ leading to explicit representations of the effective driving force $\Delta G^{\prime}$ and the dissipation energy function $\Delta G_{R}(T)$ in terms of internal friction $\left(Q^{-1}\right)$ and the shear modulus $(\mu)$. We have shown in Ref. 1 that the $Q^{-1}$ during the FOPT can be expressed as

$$
Q^{-1}=Q_{s}^{-1}+Q_{\mathrm{dyn}}^{-1}=B(T) \omega^{1-2 l}+A(T) \dot{T}^{n} / \omega^{n+2 l},
$$

where

$$
Q_{s}^{-1}=B(T) \omega^{1-2 l}
$$

and

$$
Q_{\mathrm{dyn}}^{-1}=A(T) \dot{T}^{n} / \omega^{n+2 l} .
$$

$Q_{s}^{-1}$ is the internal friction corresponding to the situation where the phase interface (PI) is stationary; we may even call $B(T)$ the stationary coefficient. $Q_{\text {dyn }}^{-1}$ gives the dynamic part of the internal friction, dependent on $\dot{T}, T$, and $\omega$, where $A(T)$ is the dynamic coefficient. These were derived supposing that the net driving force on the PI is [Eq. (3c) of Ref. 1]

$$
\Delta G^{\prime}=\Delta G_{d}-\Delta G_{R}=A_{1}(T)\left(T-T_{s}\right)^{n},
$$

where $\Delta G_{d}$ is the driving force for the PT,

$$
\Delta G_{d}=\frac{\Delta H}{T_{0}}\left(T-T_{0}\right)
$$

and $\Delta G_{R}$ is the resistance to the motion of the PI exerted by imperfections of the crystal lattice, lattice vibrations, etc.

Clearly, the coupling index $l$ is the most important parameter. It specifies the relation $Q_{s}^{-1}-\omega$ and describes the degree of response of the PI in the sample to external oscillating stress; it is a measure of the mobility of the PI involved. The index $n$ may be called the effective driving index and is a measure of the effective part of the phase transition (PT) driving force. In order to test the theory and understand further the nature of a FOPT, we have analyzed ceramic $\mathrm{VO}_{2}$ and FeMn samples in Refs. 2 and 3 and found that $l$ describes essentially the ease with which the PI vibrates during a FOPT, while $n$ actually describes the degree of effectiveness of the net driving force. In particular, the FOPT involved in $\mathrm{VO}_{2}$ is a lattice reconstructive-type leading to a noncoherent type of PI so that the distortion of the lattice structure on both sides of the PI is relatively small. ${ }^{4} \mathrm{We}$ expect that the net or effective driving force in $\mathrm{VO}_{2}$ is relatively small also. Since $n$ increases monotionically with the net driving force according to Eq. (2a), we expect $n$ to be relatively small accordingly. The value found for $n$ during the PI motion in $\mathrm{VO}_{2}$ was 0.1. Turning now to the FeMn sample in Ref. 3, the burst-type martensitic transformation (MT) there is of the displacive type, leading to a semicoher- 
ent PI. As a consequence, a large distortion of lattice structure occurs on both sides of the PI during the PT. We expect, in this case, that the net driving force and, hence, $n$ are relatively large. Indeed, we have found from analyzing our experimental data that $n=0.33$ (heating) and 0.47 (cooling), which are significantly larger than 0.1 , the value obtained for the $\mathrm{VO}_{2}$ sample. Now let us consider the values of $l$ deduced for these two samples. The FOPT in $\mathrm{VO}_{2}$ is a reconstructive type and the amount of distortion is very localized at any instant of time during PI motion. The PI could not respond sensitively to external influences such as that introduced by forced oscillations, leading to a relatively large $l$ (such as 0.2 in the $\mathrm{VO}_{2}$ sample). On the other hand, the burst-type MT in the FeMn alloy sample is a displacive type, implying that the lattice distortion is broader in space, so that the response of the PI to external influence is high and, consequently, the value of $l$ should be small, as found in Ref. 3 [0.015 (heating) and 0.035 (cooling)]. In summary, the theory leads to consequences which can fit experimental data, and the FOPT of the $\mathrm{VO}_{2}$ and $\mathrm{FeMn}$ samples can be characterized by the two indices $l$ and $n$.

There are three main objectives of writing this article.

(i) To test the theory further and understand the nature of the FOPT, we sought a sample with a displacive crystal transformation, like the FeMn sample, but which undergoes a relatively small degree of lattice distortion. The FOPT in a $\mathrm{Ni}_{50.3} \mathrm{Ti}_{49.7}$ (abbreviated NiTi) alloy falls into the category we wanted. We should note also that thermal hysteresis $\Delta T$ $=\left|T_{s}-T_{0}\right|$ for this typical NiTi sample is $\leqslant 5 \mathrm{~K}$, much smaller than that of the FeMn sample. We hope also to get some information on the influence of $\Delta T$ on the values of $l$ and $n$. The methodology to achieve this objective is similar to that in Refs. 2 and 3.

(ii) To extend the theory to the situation where the FOPT is induced by stress, rather than induced by variation of temperature.

(iii) To compare the results of the two different induction processes with similar type of samples, so far as the reversible $I / C$ PT can be identified.

In Sec. II, we develop our theory to describe the FOPT which is driven by the application of stress. We shall find that all the basic equations hold if $\dot{T}$ is replaced by $\dot{\varepsilon}$ and $\varepsilon$ is a linear function of time. A typical NiTi sample (like $\mathrm{Ni}_{49} \mathrm{Ti}_{51}$ or $\left.\mathrm{Ni}_{50.3} \mathrm{Ti}_{49.7}\right)$ with close to equal amounts of $\mathrm{Ni}$ and $\mathrm{Ti}$ does not demonstrate a linear $\varepsilon-t$ behavior. However, with doping of a tiny amount of $\mathrm{Fe}$, the $\mathrm{Ni}_{49} \mathrm{FeTi}_{50}$ (NiFeTi) sample offers a sizable domain where the $\sigma-\varepsilon$ relation is linear, leading to a $\varepsilon-t$ linear region as required. For this reason, we have used a second sample, i.e., NiFeTi for the characterization process in the stress-induced situation.

We devote Sec. III to training of the two slightly different samples and the measurements of $Q^{-1}$ as well as $\mu$. Analysis of the data is presented in Sec. IV. Relevant discussions and conclusions based on this investigation are given in the last section.

\section{EXTENSION OF THE PREVIOUS THEORY OF FOPT DRIVEN BY AN EXTERNAL STRESS}

It is well known that any specimen capable of a thermo elastic MT demonstrates the shape memory effect (SME) and
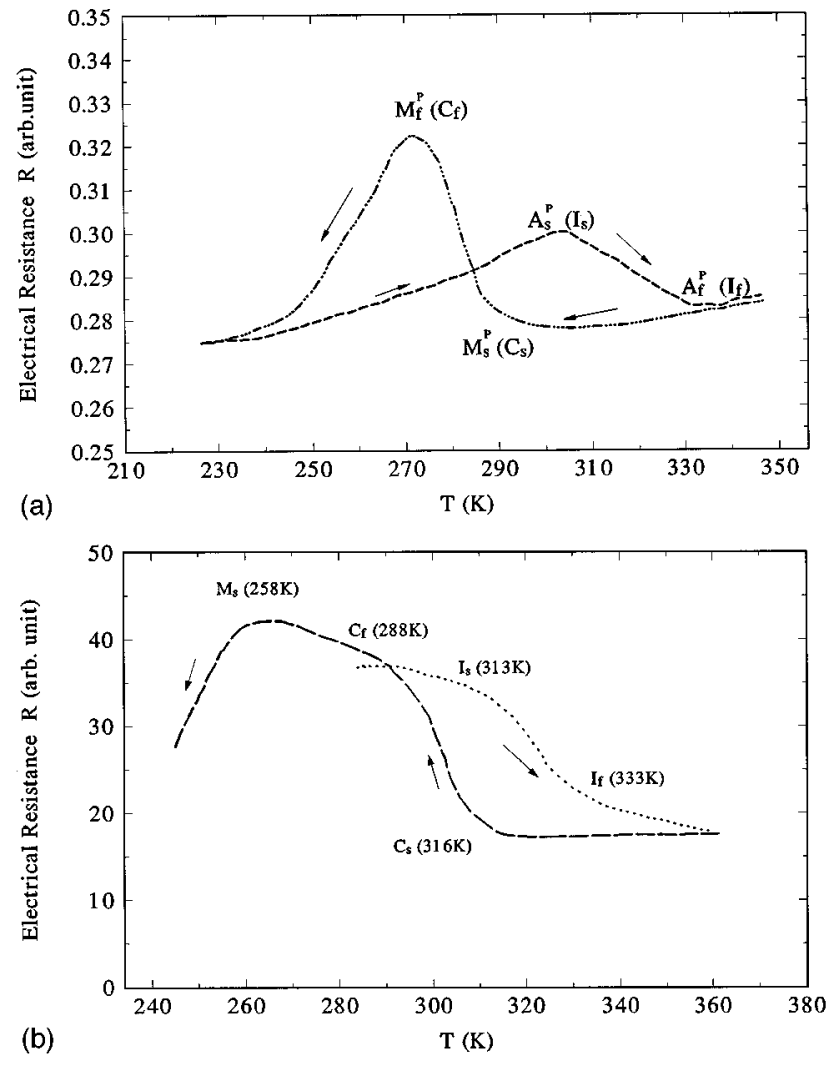

FIG. 1. (a) Variation of electrical resistance $R$ with respect to change in temperature $T$ for the $\mathrm{Ni}_{49} \mathrm{Ti}_{51}$ sample. The premartensite PT $(I / C)$ occurs in the high-temperature range 290-272 K, and the martensite PT $(C / M)$ occurs in the lower-temperature range $<272 \mathrm{~K}$. (b) Electrical resistance $R$ against temperature $T$ for the NiFeTi sample. Note that the $I / C$ and $C / M$ PT's are well separated in temperature $(316-288 \mathrm{~K}$, below $258 \mathrm{~K})$ with the introduction of Fe doping in this sample.

pseudoelasticity (PE). The SME is provided by the thermoelastic MT induced by temperature variation; the PE is supplied also by the thermo elastic MT, but induced by stress. ${ }^{5-8}$ In the previous two examples, the PT has been induced by variation of temperature and we purposely induce a PT by applying an external stress in this paper. Thus we focus our analysis on the pseudoelastic properties in this paper, providing a different (stress-induced PT) method to test the theory. In actual experimentation, we applied the external stress at a temperature (at or slightly higher than) $A_{f},{ }^{8-10}$ we then studied the relation between the consequential PT strain and $Q^{-1}$. We also analyzed the $Q^{-1}$ - $\omega$ behavior. When we measured the electrical resistance of a MT sample as a function of temperature, we obtain two typical types of curves. In Fig. 1(a), a pre martensite PT occurs within the temperature range $M_{s}^{P}-M_{f}^{P}\left(C_{s}-C_{f}\right)$ for the $\mathrm{Ni}_{49} \mathrm{Ti}_{51}$ sample; here, the superscript $P$ indicates that the $\mathrm{PT}$ within the range $M_{s}^{P}-M_{f}^{P}$ is pre martensitic. Below the temperature $M_{f}^{P}\left(C_{f}\right)$, the MT takes place and we may take $M_{s} \approx M_{f}^{P}\left(C_{f}\right)$ in this sample. In the second situation of the NiFeTi sample, $M_{s}$ is significantly lower than $M_{f}^{P}\left(C_{f}\right)$ and a corresponding $R-T$ curve is shown in Fig. 1(b). Note that the $I / C$ and $C / M$ PT's are separated in the second situation of the NiFeTi sample. Since we plan to analyze the $I / C \mathrm{PT}$, we need a sample showing that the $I / C$ and $C / M$ PT's are well separated in temperature. For 

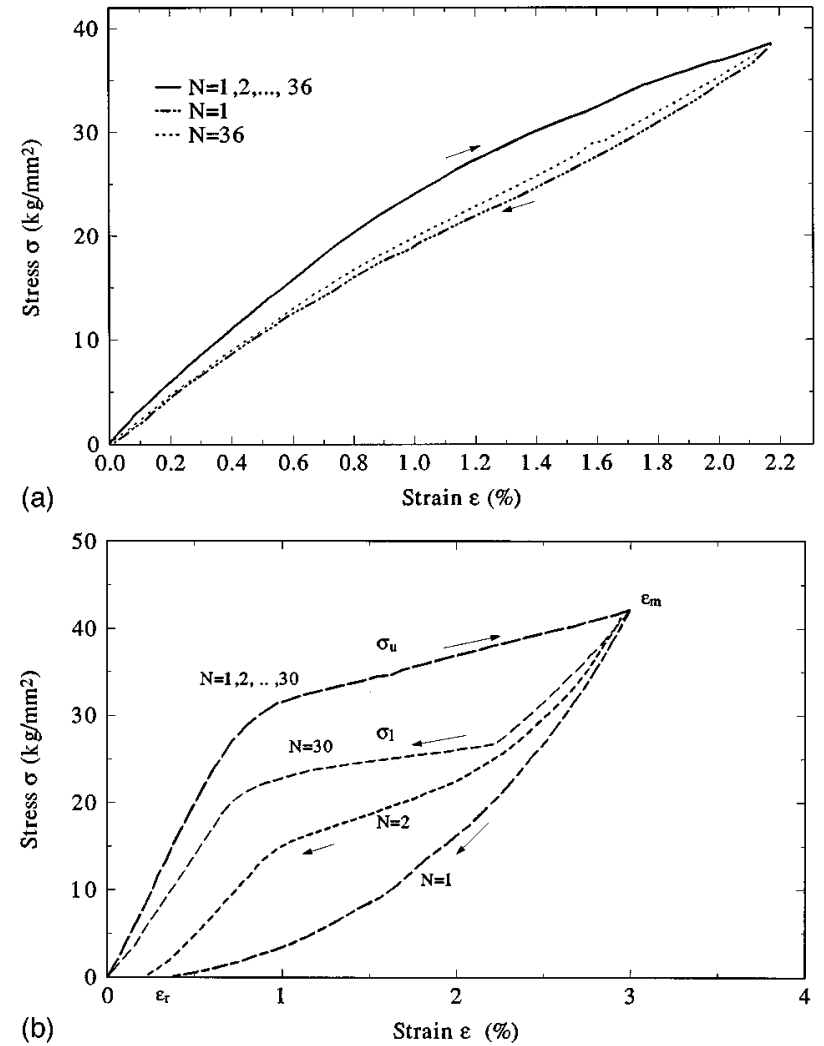

FIG. 2. (a) Typical stress $\sigma$ versus strain $\varepsilon$ for the NiTi alloy with almost equal amounts $\left(\mathrm{Ni}_{49} \mathrm{Ti}_{51}\right)$ during training at $T$ $=298 \mathrm{~K}$, near $M_{S}^{P}$. Here $N$ specifies the result after the $N$ th training cycle. (b) Stress $\sigma$ vs strain $\varepsilon$ for the NiFeTi sample during training at $T=333 \mathrm{~K}$. Here $N$ signifies the result after the $N$ th training process.

this reason, we have used a NiFeTi sample.

Figure 2(a) demonstrates a typical "shuttle-type" stressstrain loop of the NiTi sample during a stress-induced thermo elastic FOPT in a trained polycrystalline PE sample; the training method is described in Sec. III B. The upper curve was taken during loading. The stress $\sigma$ is decreased by reducing the load and the $\sigma_{l^{-}} \varepsilon$ curve does not pass through the origin after the first stress cycle. Generally, after a few tens of cycles, we have a loop closed at $\varepsilon=0$ and $\sigma=0$ as shown in Fig. 2(a). The driving force $\Delta G_{d}$ for the stressinduced thermoelastic MT is ${ }^{8,9,11}$

$$
\Delta G_{d}=\frac{\varepsilon_{0}}{2}\left(\sigma_{u}-\sigma_{l}\right)
$$

Of course, the $\sigma-\varepsilon$ relation holds at or around the temperature $A_{f}$ in Fig. 1(a). Such a loop, as indicated in Fig. 2(a), pertains to the situation where there is mainly one new $M$ phase during the PT. Note that a large temperature gap $\left[M_{s}^{P}\left(C_{s}\right)-M_{s}\right] \sim 30-50 \mathrm{~K}$ appeared in our NiFeTi specimen as indicated in Fig. 1(b), enabling us to analyze the $I / C$ PT by itself. The corresponding $\sigma-\varepsilon$ loop (called a type-II thermoelastic PT) appears like that shown in Fig. 2(b), with "four straight sides and a tail." In this type-II situation, $\left(\sigma_{u}-\sigma_{l}\right)$ varies linearly with $\varepsilon$. Then $\Delta G_{d}$ can be expressed as

$$
\Delta G_{d}=\frac{\varepsilon_{0}}{2}\left(\sigma_{u}-\sigma_{l}\right)=A_{0} \varepsilon=A_{0} \dot{\varepsilon} t
$$

in the situation of loading with a constant $\dot{\varepsilon}$, where $A_{0}$ is a constant. Equation (4) has the same form as Eq. (2) in Ref. 1. Following our analysis in Sec. II of Ref. 1, we have the following explicit form for the effective driving force:

$$
\Delta G^{\prime}=\Delta G_{d}-\Delta G_{R}=A_{1}(\varepsilon)(\dot{\varepsilon} t)^{n} .
$$

Equation (5) is identical to relation (3c) in Ref. 1, implying that the solution for the equation of motion of the PI is the same as that depicted in Eq. (1), substantiating the applicability of our theory to the stress-induced PT at constant temperature and constant $\dot{\varepsilon}$, i.e.,

$$
Q^{-1}=B(\varepsilon) \omega^{1-2 l}+A(\varepsilon) \dot{\varepsilon}^{n} / \omega^{n+2 l} .
$$

It is well established that the premartensitic PT is an independent transformation preceding the normal martensitic PT in a NiFeTi alloy. ${ }^{5-13}$ During a cooling process, the NiFeTi alloy undergoes a second-order PT at first and the parent phase $(P, B 2, \mathrm{CsCl})$ transforms to an incommensurate phase $(I, B 2)$, which then transforms to a commensurate phase ( $C$, rhombohedral); the last PT is a first-order one. Both of the $P / I$ and $I / C$ transitions in NiFeTi alloy fall into the category of premartensitic PT, but the $I / C$ PT can demonstrate the SME and PE.

The investigation of the PE effect using the $Q^{-1}$ method during a process of stress-induced PT was initiated in the 1980. ${ }^{10,14,15}$ A $Q^{-1}$ peak and a minimum of $\mu$ were observed in the $Q^{-1}-\varepsilon$ and $\mu-\varepsilon$ curves, respectively. The peak height of the $Q^{-1}-\varepsilon$ curve, i.e., $Q_{P}^{-1}$, and the minimum of $\mu$ in the $\mu-\varepsilon$ graph were found to be more pronounced with increasing strain rate $\dot{\varepsilon}$ and decreasing frequency $\omega$. The concept of dynamics of the PI was used then to explain such experimental findings. ${ }^{10,15,16}$ More recently, Stoiber et al. ${ }^{17}$ have introduced a technique of $Q^{-1}$ measurement to investigate the $\beta_{1} / \beta_{1}^{\prime}$ interface motion during the stress-induced MT in $\mathrm{Cu}-\mathrm{Zn}$-Al single crystals. They made a "window" in parallelepiped specimen, a PI was introduced on a cross section of the parallelepiped sample, and a $Q^{-1}$ peak was observed during loading and unloading. The shear stress induced by torsion had a vector component along the PI (according to Ref. 1, this is just the condition for the occurrence of coupling between a PI and oscillating stress), and a $Q^{-1}$ peak was observed in the $Q^{-1}-\sigma$ graph - such a condition was believed to be satisfied in their sample with "window." The experimental finding is very interesting, and they concluded that " the majority of the energy dissipation during single variant transformations is due to the nucleation process and not due to the growth of the new phase."

\section{EXPERIMENTATION AND EXPERIMENTAL RESULTS}

\section{A. Sample training and measurements for the $\mathrm{Ni}_{50.3} \mathrm{Ti}_{49.7}$ specimen}

The NiTi alloys are best known for demonstrating the SME which is provided by the thermo elastic MT induced by temperature variation. ${ }^{5-8}$ More than one type of the thermoelastic MT (induced by temperature or stress) can occur in 


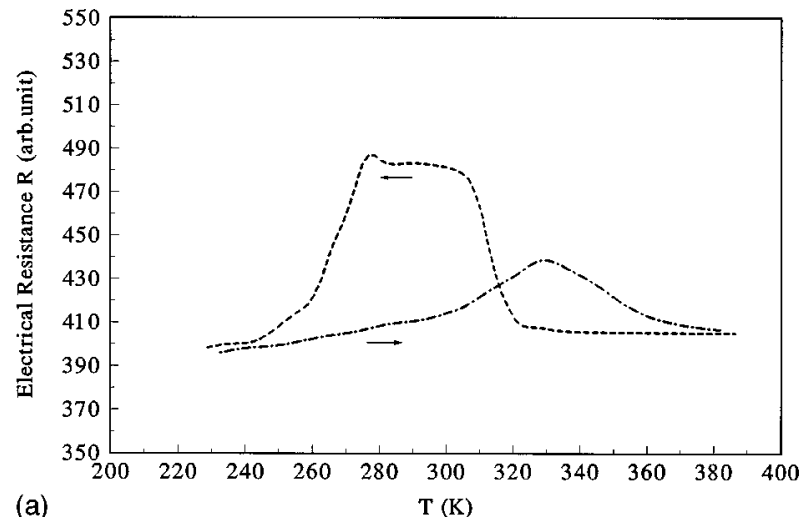

(a)

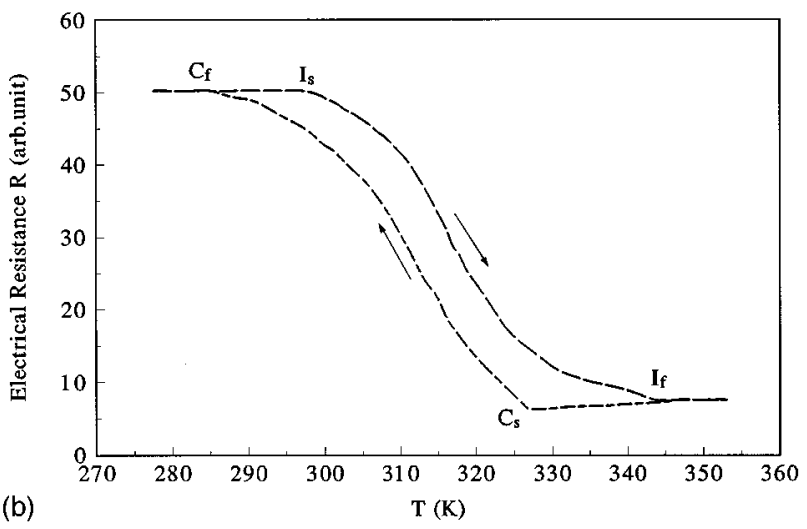

FIG. 3. (a) $R-T$ curve of the NiTi sample after the constraint aging process. (b) $R-T$ curve of the NiTi sample after ten cycles of training. An $I / C$ PT appeared within the temperature band of 280$350 \mathrm{~K}$.

most alloys which demonstrate the SME, including NiTi alloys. ${ }^{13,18-20}$ In particular, in the set of NiTi alloys with various compositions, there are two thermoelastic MPT's during cooling, one from incommensurate ( $I$ or $B 2$ phase) to commensurate $(C$, rhombohedral or $\mathcal{R}$ phase), and the other from commensurate to the martensitic phase with a $B 19$ structure. It should also be noted that the relative amounts of the $I, C$, and $M$ phases in the sample are very sensitive to the thermomechanical history. ${ }^{21-25}$ In this paper, we focus on reversible $I / C$ transitions after introducing a special training process to separate the $I / C$ transition from the $C / M$ transition. With just the $I / C$ transition, we can proceed to test the theory.

The wire sample, with nominal composition $\mathrm{Ni}_{50.3} \mathrm{Ti}_{49.7}$ (at. \%) of diameter $1.0 \mathrm{~mm}$ and length $50 \mathrm{~mm}$, was supplied by the General Research Institute of Nonferrous Metals, Beijing, China.

Two-way-shape-memory training is necessary to ensure that the $I / C$ transformation is completely reversible and to separate the $I / C$ transformation from the $C / M$ PT. We have employed the constraint aging ${ }^{26,27}$ (CA) method.

The wire was (a) first annealed at $993 \mathrm{~K}$ for $1 \mathrm{~h}$ followed by an ordinary air cooling process, then (b) heated at $673 \mathrm{~K}$ for $1 \mathrm{~h}$, followed by furnace cooling, and then (c) stretched by $\varepsilon=2.5 \%$, while being held at $393 \mathrm{~K}$. The stretched sample was clamped at both ends to maintain constant length, heated to $673 \mathrm{~K}$ for $1 \mathrm{~h}$, and then furnace cooled to room temperature. The $R-T$ curve after the process of $\mathrm{CA}$ is shown in Fig. 3(a). Further thermocycles ( $N>10$ times) were

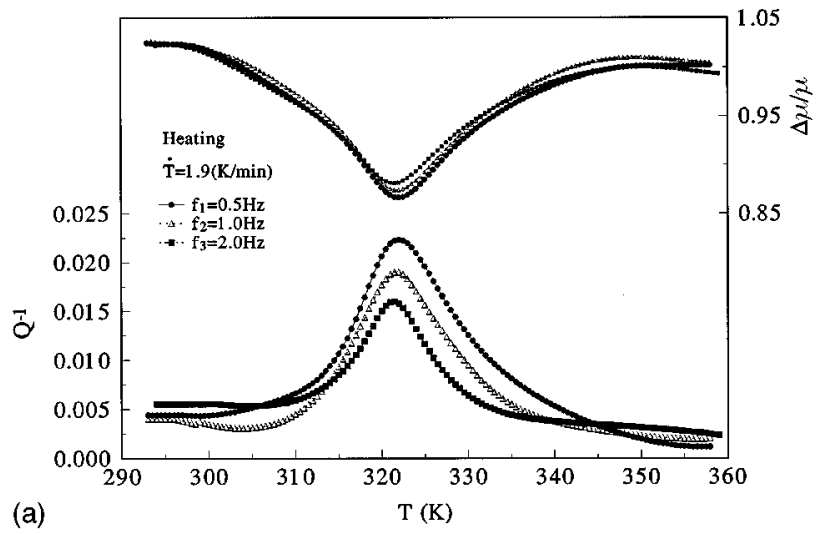

(a)

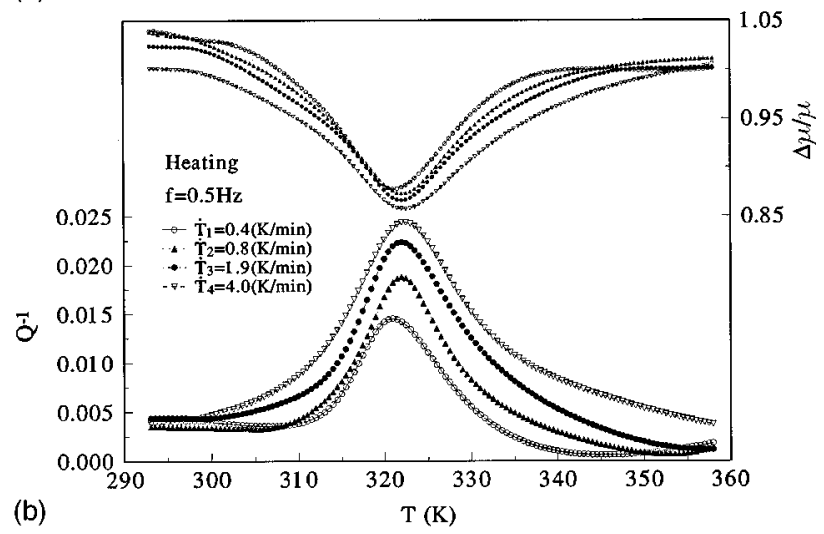

FIG. 4. (a) $Q^{-1}$ and $\mu$ against $T$ for the NiTi sample during heating with $\dot{T}=1.9 \mathrm{~K} / \mathrm{min}$ for different $f: f_{1}=0.5 \mathrm{~Hz}, f_{2}$ $=1.0 \mathrm{~Hz}$, and $f_{3}=2.0 \mathrm{~Hz}$. (b) $Q^{-1}$ and $\mu$ vs $T$ for the NiTi sample during heating with $f=0.5 \mathrm{~Hz}$ for different $\dot{T}: \dot{T}_{1}=0.4 \mathrm{~K} / \mathrm{min}$, $\dot{T}_{2}=0.8 \mathrm{~K} / \mathrm{min}, \dot{T}_{3}=1.9 \mathrm{~K} / \mathrm{min}$, and $\dot{T}_{4}=4.0 \mathrm{~K} / \mathrm{min}$.

carried out in the temperature range from 273 to $353 \mathrm{~K}$, separating the temperature ranges of the PT's: $M / C$ from $C / I$. The sample was then ready for use.

The electrical resistance of the trained sample, for the range from room temperature to $365 \mathrm{~K}$, Fig. 3(b), shows clearly that a $I / C$ PT has occurred. Moreover, with x-raydiffraction analysis, we identified a reversible $I / C$ PT within the temperature range of $280-350 \mathrm{~K}$. The experimental result also showed that if the wire was not trained, the $M$ phase on heating changed to a mixture of $M$ phase and $C$ phase around $328 \mathrm{~K}$ and then to $I$ phase at $393 \mathrm{~K}$ during heating. On cooling, the usual $I-C-M$ phase transitions occurred, but the resistance peak was rather narrow without a plateau and the nature of the various phase transitions is the same as that in Fig. 3(a). However, after training, only an $I / C$ phase transformation occurred [as in Fig. 3(b)].

The $Q^{-1}$ of the sample was measured by a vacuum inverted torsion pendulum as in Ref. 3. The free decay mode was used for $Q^{-1}$ measurement with a maximum amplitude of $1 \times 10^{-5}$. The resolution for $Q^{-1}$ measurement is $1 \%$, and the $\mu$ could be recorded with a precision of $0.2 \%$. The $Q^{-1}$ and $\mu$ were measured simultaneously during a heating process (from room temperature to $373 \mathrm{~K}$ ) for constant $\dot{T}$ $=1.9 \mathrm{~K} / \mathrm{min}$, but for various frequencies $f_{1}=0.5 \mathrm{~Hz}, f_{2}$ $=1.0 \mathrm{~Hz}$, and $f_{3}=2.0 \mathrm{~Hz}$; these results are presented in Fig. 4(a). Also, at a fixed initial frequency $f=0.5 \mathrm{~Hz}$, we varied $\dot{T}\left(\dot{T}_{1}=0.4 \mathrm{~K} / \mathrm{min}, \dot{T}_{2}=0.8 \mathrm{~K} / \mathrm{min}, \dot{T}_{3}=1.9 \mathrm{~K} / \mathrm{min}\right.$, and $\dot{T}_{4}$ 
$=4.0 \mathrm{~K} / \mathrm{min}$ ) and recorded the $Q^{-1}-T, \mu-T$ graphs in Fig. 4(b). We also repeated the measurements of $Q^{-1}-T, \mu-T$ relations with other frequencies $(0.5,1.0$, and $2.0 \mathrm{~Hz})$ during heating, but these graphs are not shown here. Moreover, similar data were collected on cooling.

We should note that $Q^{-1}$ is defined theoretically as

$$
Q^{-1}=\frac{1}{2 \pi} \frac{\Delta w}{w},
$$

where $\Delta w$ and $w$ are the energy dissipated over one cycle and the maximum energy stored in that cycle, respectively. In experimentation, we measured the logarithmic decrement $Q_{\delta}^{-1}$ of the torsion vibration of the sample. $Q^{-1}$ is found to be related to $Q_{\delta}^{-1}$ by $^{3}$

$$
Q^{-1}=\frac{1}{2 \pi}\left[1-\exp \left(-2 \pi Q_{\delta}^{-1}\right)\right],
$$

and we have plotted the value of $Q^{-1}$ (rather than $Q_{\delta}^{-1}$ ) in all our graphs.

Comparing Figs. 4(a) and 4(b) with Fig. 3(b), we observe that a clear $Q^{-1}$ peak and a minimum in $\mu$ occur over the same range of temperature as the $I / C \mathrm{PT}$. The peak height $Q_{p}^{-1}$ increases with decreasing $f$ and increasing $\dot{T}$, while the minimum of the $\mu-T$ curve becomes more prominent as $f$ decreases and as $\dot{T}$ increases. Since the difference in the minimum of $\mu$ (i.e., $\Delta \mu$ ) can be determined, we can use the method of Ref. 2 for the $\mathrm{VO}_{2}$ sample to calculate the value of $l$ for this NiTi sample.

\section{B. Sample preparation and measurements for the $\mathrm{Ni}_{49} \mathrm{FeTi}_{50}$ specimen}

The large plate sample with nominal composition $\mathrm{Ni}_{49} \mathrm{FeTi}_{50}$ of thickness $1.3 \mathrm{~mm}$ was supplied by the General Research Institute of Non-ferrous Metals, Beijing, China. The plate was cold rolled to a thickness of $0.7 \mathrm{~mm}$ and a strip of size $0.7 \times 0.7 \times 150 \mathrm{~mm}^{3}$ was cut for our experimentation.

The four graphs, namely, $\sigma-\varepsilon, Q^{-1}-\varepsilon, \mu-\varepsilon$, and $R-\varepsilon$, were measured simultaneously using the Tensile Testing Machine (model DL-1000) manufactured by Chang Chun Tensile Testing Machine Factory. When we performed the usual tensile test, we clamped one end of the sample and loaded the other end. We attached a horizontal vibrating rod to the middle part of the sample so that the free torsional vibrations of the sample could be set up, with it its own natural frequency of free vibrations (so-called "middle torsion pendulum"). In order to excite and detect the vibrations, two standard electronic coils were fixed close to the ends of the vibrating rod, and the $Q^{-1}$ as well as the $\mu$ were calculated through the measurement of the amplitude and the frequency of vibration with an $x-y$ recorder and a millisecond meter. Two probes were attached to each end of the sample, and the standard four-probe method was employed to record the electrical resistance over the strain demonstrated. With this setup, the stated four curves were recorded by $x-y$ recorders simultaneously. Since the true length of the sample was extended and the cross section decreased accordingly during loading in the tensile test, we had to modify the shear modulus and electrical resistance to reflect the changes in geo-

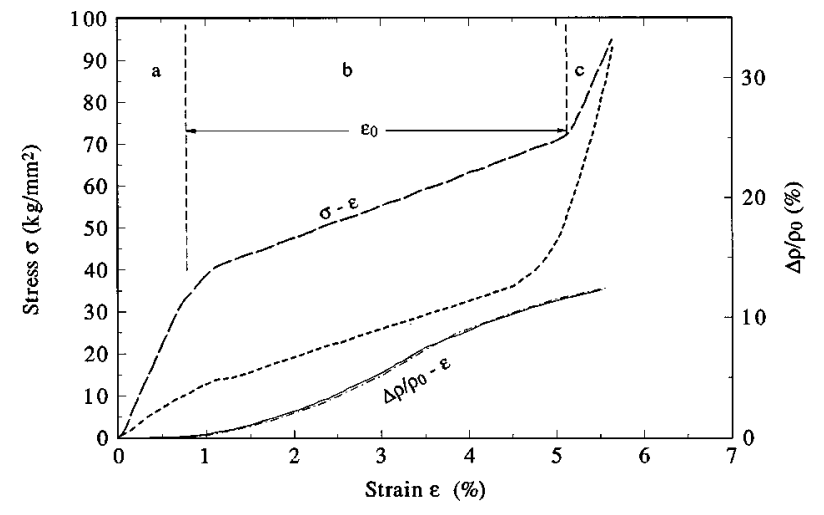

FIG. 5. Stress $\sigma$ vs strain $\varepsilon$ for the NiFeTi sample after 30 training cycles, with $\varepsilon$ extended to $5.6 \%$. Note that only one PT, i.e., $I / C$, occurs within the range $0.75 \% \leqslant \varepsilon \leqslant 4.35 \%$. The shaded area $\Delta S$ measures the enthalpy of the stress-induced $I / C$ PT.

metrical dimensions. The formula $\mu \propto f^{2}(1+\varepsilon)^{3}$ was adopted as in Ref. 14, while the relative variation in electrical resistivity was modified according to $\Delta \rho / \rho=(R$ $\left.-R_{0}\right) /\left[R_{0}(1+\varepsilon)^{2}\right]$, where $R_{0}$ is the initial resistance. ${ }^{14,28}$ Our equipment allowed a resolution of $0.1 \mathrm{~kg} / \mathrm{mm}^{2}$ for the stress measurement, while we could measure $R$ to an accuracy of $0.1 \mathrm{~m} \Omega$. The resolution for $Q^{-1}$ measurement was found to be $1 \%$, and the crystal structure analysis was carried out with the help of an x-ray diffractometer (model D/maxIIIA).

The specimen treatment began by tightly clamping it in a stainless steel holder, heating to $673 \mathrm{~K}$ for $1 \mathrm{~h}$, and then air cooling. The resistance-temperature curve during a cycle of heating and cooling is shown in Fig. 1(b). From 283 to 313 $\mathrm{K}$, the structure of the sample was rhombohedral $(C)$. When the temperature was increased from 313 to $333 \mathrm{~K}$, the specimen changed to the $B 2$ structure $(I)$ and stayed in the same form when heated further to $363 \mathrm{~K}$. In cooling from $363 \mathrm{~K}$, the specimen was in the incommensurate phase down to a temperature of $316 \mathrm{~K}\left(C_{s}\right)$, at which a commensurate phase appeared. At $288 \mathrm{~K}\left(C_{f}\right)$, the transition was completed. Further down at $258 \mathrm{~K}$, the martensite PT appeared, with a temperature gap of $30 \mathrm{~K}$ between $C_{f}$ and $M_{s}$. The sample was heated back to $333 \mathrm{~K}$, at which the training process was introduced. Figure 2(b) indicates that after the first stressstrain training cycle $(N=1)$, there was a residue strain of $\varepsilon_{r} \approx 0.4 \%$ when $\sigma$ was reduced to zero. After 30 cycles of training, the $\sigma$ - $\varepsilon$ loop maintained the same shape with $\varepsilon \rightarrow 0$ as $\sigma \rightarrow 0$ during unloading. In this training process, the maximum strain reached was kept to $\varepsilon_{m}=3 \%$.

In order to find the maximum $\varepsilon_{m}$ reached by the strain of the $I / C$ PT which left the $\sigma-\varepsilon$ loop form closed and practically unchanged, we used the other sample and tried $\varepsilon_{m}$ $=6 \%$ and $7 \%$ for 30 training cycles using two very similarly prepared samples. We have found that if $\varepsilon_{m}$ was extended to $7 \%$, the residual $\varepsilon_{r}$ was not equal to zero, while in the case $\varepsilon_{m}=5.6 \%$, the $\sigma-\varepsilon$ loop was kept very close to constant (see Fig. 5). From this figure we see that the maximum strain $\varepsilon_{0}$ supplied by $I / C$ transition was found to be $4.35 \%$ and the steep rise of the $\sigma-\varepsilon$ curve for $\varepsilon>5.0 \%$ represents a transition contributed by a martensitic transformation.

Further experimentation on the $\sigma-\varepsilon$ relations showed that, when $\varepsilon_{m}>3 \%$, the quantity of residual $M$ (or sometimes 

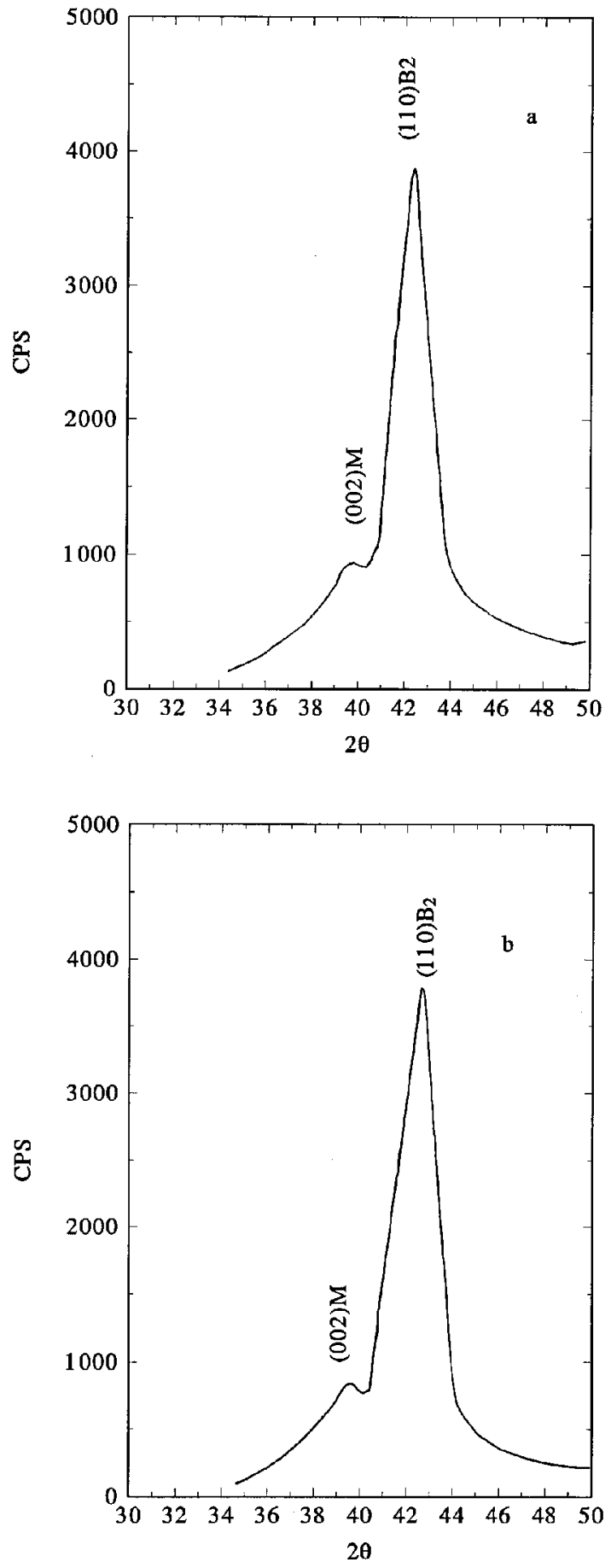

FIG. 6. (a) Relative intensity of x-ray diffraction as measured by the number of counts per sound (CPS) of the NiFeTi specimen before the 30th training cycle for the diffraction angle of $2 \theta$ ranging from $30^{\circ}$ to $50^{\circ}$ at $T=333 \mathrm{~K}$. (b) Relative intensity against $2 \theta$ corresponding to (a) after the 30 th training cycle at $T=333 \mathrm{~K}$.

$C$ ) phase varied on performing each cycle. Only when $\varepsilon_{m}$ $<3 \%$ was the amount of residual $M$ phase relatively small and constant after each cycle. We therefore characterized and analyzed our results based on the $\sigma-\varepsilon$ relation corresponding to $\varepsilon_{m}=2.5 \%$, making sure that the $I / C$ PT was reversible and the amount of residue $M$ phase unvaried during the stress-induced PT. We recorded the relative intensities of the $\mathrm{X}$-ray-diffraction patterns for a range of diffraction angle $2 \theta$ before and after the 30th training cycle in order to carry out
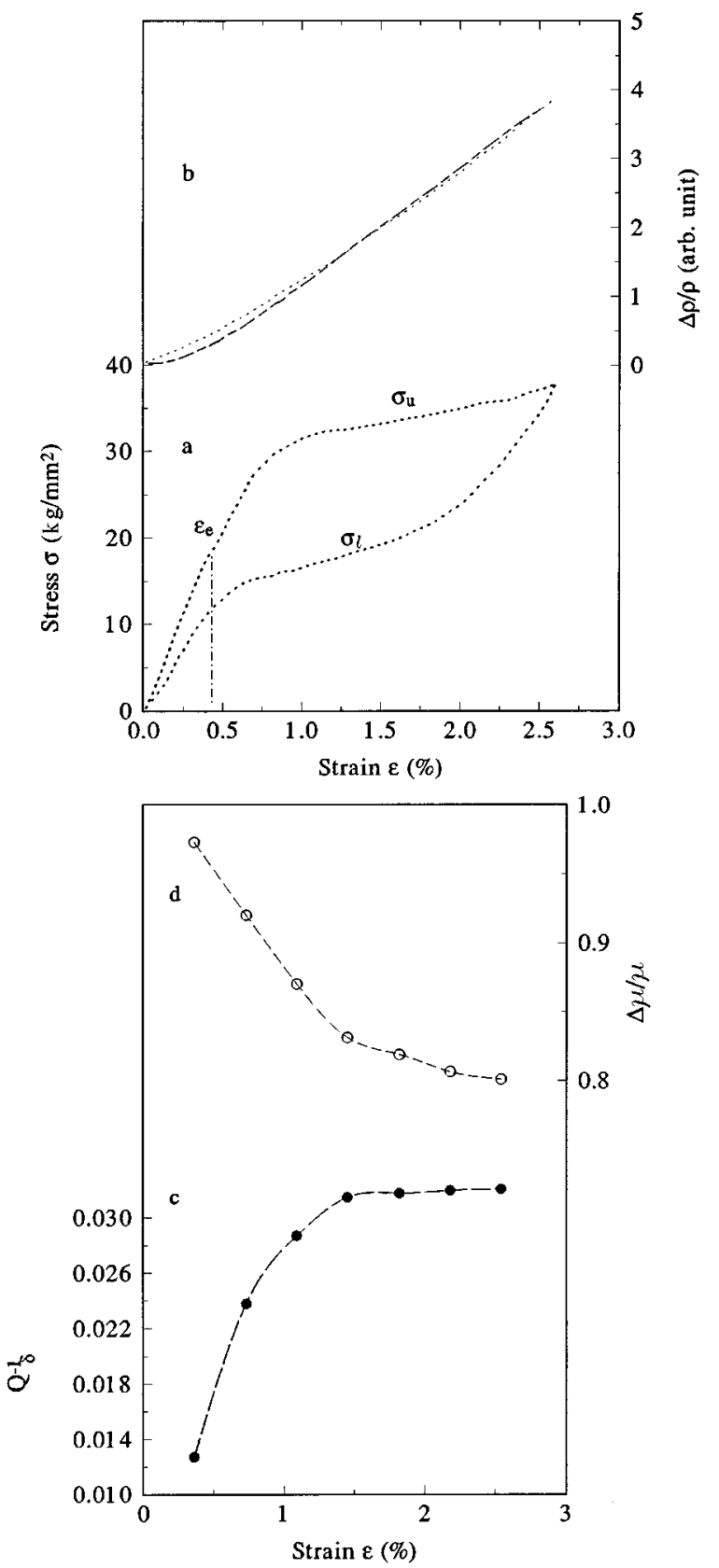

FIG. 7. (a) Stress $\sigma$ vs strain $\varepsilon$ for the trained NiFeTi sample. (b) Relative resistivity $\Delta \rho / \rho$ against strain $\varepsilon$ for the NiFeTi sample. (c) $Q^{-1}$ versus strain $\varepsilon$ for the NiFeTi sample. (d) Relative shear modulus $\mu\left(f^{2} / f_{0}^{2}\right)$ against strain $\varepsilon$. The strain rate $\dot{\varepsilon}_{2}=9.0$ $\times 10^{-5} \mathrm{~s}^{-1}$, the temperature $T=333 \mathrm{~K}$ and the oscillation frequency $f_{4}=2.5 \mathrm{~Hz}$ are fixed for (a)-(d).

phase identification [see Figs. 6(a) and 6(b)]. Comparing Figs. 6(a) and 6(b), we clearly observe that there are the same two phases in both situations-the dominant $I$ phase and a small residue $M$ phase.

The effect of changing the strain rate $\dot{\varepsilon}$ on the $Q^{-1}$ characteristics during a stress-induced $I / C$ transition was investigated with three constant strain rates $\dot{\varepsilon}$, namely, $\dot{\varepsilon}_{1}=3.0$ $\times 10^{-5} / \mathrm{s}, \dot{\varepsilon}_{2}=9.0 \times 10^{-5} / \mathrm{s}$, and $\dot{\varepsilon}_{3}=21.3 \times 10^{-5} / \mathrm{s}$. The influence of the measuring frequency was measured by five different initial frequencies: $f_{1}=0.6 \mathrm{~Hz}, f_{2}=0.9 \mathrm{~Hz}, f_{3}$ $=1.5 \mathrm{~Hz}, f_{4}=2.5 \mathrm{~Hz}$, and $f_{5}=3.6 \mathrm{~Hz}$ for each strain rate 


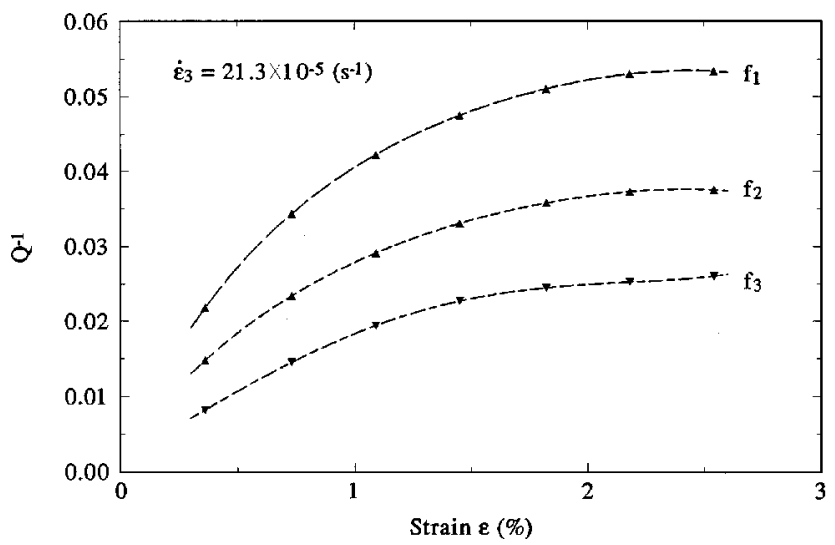

FIG. 8. $Q^{-1}$ against strain $\varepsilon$ for three frequencies $f_{1}=0.6 \mathrm{~Hz}$, $f_{3}=1.5 \mathrm{~Hz}$, and $f_{5}=3.6 \mathrm{~Hz}$, all at $\dot{\varepsilon}_{3}=21.3 \times 10^{-5} \mathrm{~s}^{-1}$ and $T$ $=333 \mathrm{~K}$ (NiFeTi sample).

$\dot{\varepsilon}$ mentioned above. $Q^{-1}$ during the PT is independent of the strain amplitude of oscillation.

The variation with $\varepsilon$ of $\sigma$, the change in relative resistivity $\Delta \rho / \rho, Q^{-1}$, and $\mu$ during the stress-induced $I / C$ transition for the trained $\mathrm{NiFeTi}$ sample are plotted with respect to change in strain $\varepsilon$ in Figs. 7(a) $-7($ d), respectively; the strain rate $\dot{\varepsilon}_{2}=9.0 \times 10^{-5} / \mathrm{s}$ and $f_{4}=2.5 \mathrm{~Hz}$ are constant during these measurements. It is clear from Figs. 7(a) and 7(b) that the $\sigma-\varepsilon$ and $\Delta \rho / \rho-\varepsilon$ loops are closed at $\sigma=0$ and $\varepsilon=0$. Here $Q^{-1}$ increases, but $\mu$ decreases with increasing $\varepsilon$ as revealed in Figs. 7(c) and 7(d), respectively. The existence of two practically linear sections in both $\sigma_{l^{-}} \varepsilon$ and $\sigma_{\mu^{-}} \varepsilon$ curves of Fig. 7(a) demonstrates that the dissipation theory proposed in Ref. 1 can be applied to the analysis of the dissipative behavior during the stress-induced $I / C$ transition in this particular NiFeTi alloy.

Figure 8 shows the variation of $Q^{-1}$ with $\varepsilon$ for three different frequencies as marked and at a constant strain rate $\dot{\varepsilon}_{3}=21.3 \times 10^{-5} / \mathrm{s}$. For three measuring strain rates $\dot{\varepsilon}$ as labeled and at a constant frequency $f_{1}=0.6 \mathrm{~Hz}$, we have the $Q^{-1}-\varepsilon$ plots shown in Fig. 9. Note that $Q^{-1}$ increases with increasing $\dot{\varepsilon}$, but decreases with increasing frequency.

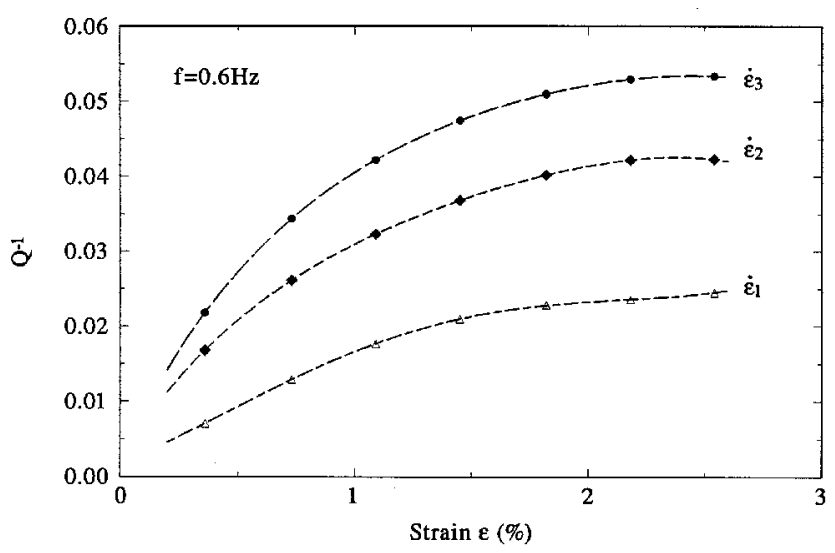

FIG. 9. $Q^{-1}$ vs percentage strain $\varepsilon$ for three strain rates $\dot{\varepsilon}_{1}$ $=3.0 \times 10^{-5} \mathrm{~s}^{-1}, \dot{\varepsilon}_{2}=9.0 \times 10^{-5} \mathrm{~s}^{-1}$, and $\dot{\varepsilon}_{3}=21.3 \times 10^{-5} \mathrm{~s}^{-1}$ all for $f_{1}=0.6 \mathrm{~Hz}$ and at $T=333 \mathrm{~K}$ (NiFeTi sample).

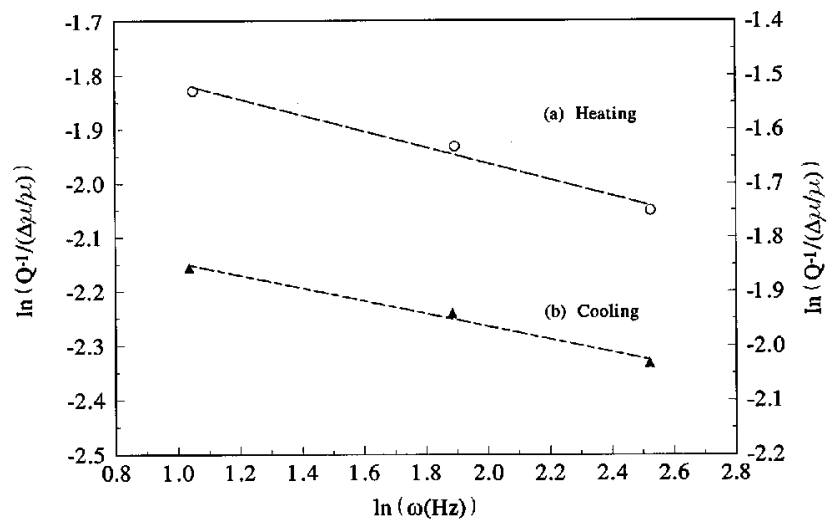

FIG. 10. $\ln \left[Q^{-1} /(\Delta \mu / \mu)\right]$ against $\ln [\omega(\mathrm{Hz})]$ during heating and cooling for the NiTi sample.

\section{DATA ANALYSIS}

\section{A. Temperature-induced thermoelastic martensitic transformation}

In a phase transformation study, we usually estimate the fractional volume $F$ of the new phase at a particular $T$ by measuring the fractional change in electrical resistance at that temperature. We can see from Fig. 3(b) the temperature $T_{1 / 2}$ at which the resistance is just half of the highest value. Note that $T_{1 / 2}$ depends on $\dot{T}$ also, but is independent of frequency $\omega$, a result obtained in Ref. 2 and which is assumed to be valid in the present study. For this particular $\dot{T}$, we obtain the $Q_{1 / 2}^{-1}$ and $\mu_{1 / 2}$ values at this $T_{1 / 2}$ [from Fig. 4(b), corresponding to heating].

We now proceed to evaluate the crucial indices $l$ and $n$. Note that the relative shear modulus defect $\Delta \mu / \mu$ consists of two parts: ${ }^{1}(\Delta \mu / \mu)_{S M}$ corresponds to the softening of the phonon mode (pertaining to $\dot{T} \rightarrow 0$ and independent of $\dot{T}$ ), and the other part $(\Delta \mu / \mu)_{\mathrm{PI}}$ is contributed by the motion of the PI (corresponding to $\dot{T} \neq 0$ ).

Using the method described in detail previously ${ }^{2}$ and using data points obtained from the whole set of experimental results like that in Figs. 4(a) and 4(b), we have plotted lines for heating (a) and cooling (b) in Fig. 10, from the slopes of which we obtain $l=0.15$ (heating) and 0.12 (cooling), which are also listed in Table I. Having found $l$, we can now use Eq. (1) in the form

$$
Q_{1 / 2}^{-1} /\left(\omega^{1-2 l}\right)=B\left(T_{1 / 2}\right)+A\left(T_{1 / 2}\right) \dot{T}^{n} /\left(\omega^{1+n}\right),
$$

to obtain the values of $n$. We now take $n$ as a parameter and for a series of values of $n$ plot $Q_{1 / 2}^{-1} / \omega^{0.7}$ (for heating) against $\dot{T}^{n} /\left(\omega^{1+n}\right)$ with the set of $Q_{1 / 2}^{-1}$ data. The values of $n$ that minimize the error are $n=0.35$ (for heating) and $n=0.30$ (for cooling). Figure 11 gives two graphs of $Q_{1 / 2}^{-1} /\left(\omega^{1-2 l}\right)$ versus $\dot{T}^{n} /\left(\omega^{1+n}\right)$, during heating and cooling; they are close to straight lines, leading to a determination of the indices $n$ and $l$ as listed in Table $\mathrm{I}$, along with the values of $n$ and $l$ for $\mathrm{VO}_{2}$ (Ref. 2) and FeMn (Ref. 3).

\section{B. Stress-induced thermoelastic martensitic transformation}

For the second sample, NiFeTi, using data points obtained from the whole set of experimental results like those pre- 
TABLE I. Indices $n$ and $l$ of several samples during FOPT.

\begin{tabular}{|c|c|c|c|c|c|}
\hline & \multicolumn{2}{|c|}{$n$} & \multicolumn{2}{|c|}{$l$} & \multirow{2}{*}{$\begin{array}{c}l_{0} \\
\text { Step }(\dot{T}=0)\end{array}$} \\
\hline & Heating & Cooling & Heating & Cooling & \\
\hline $\mathrm{VO}_{2}$ & 0.1 & & 0.2 & & \\
\hline $\mathrm{Fe}-18.8 \% \mathrm{Mn}$ & 0.33 & 0.47 & 0.06 & 0.02 & 0.86 \\
\hline $\mathrm{Ni}_{50.3} \mathrm{Ti}_{49.7}$ & 0.35 & 0.30 & 0.15 & 0.12 & \\
\hline \multirow{2}{*}{$\mathrm{Ni}_{49} \mathrm{FeTi}_{50}$} & & stretching & & stretching & \\
\hline & & $0.35-0.38$ & & $0.12-0.14$ & \\
\hline
\end{tabular}

sented in Figs. 8 and 9, we can plot $\ln Q^{-1}$ against $\ln \omega$ in Fig. 12(a) for three fixed strain rates $\dot{\varepsilon}_{1}, \dot{\varepsilon}_{2}$, and $\dot{\varepsilon}_{3}$ stated before, while the specimen contains $46 \%$ of $C$ phase. We refer to Fig. 7(a) for the estimation of the "background internal friction" $Q_{b}^{-1}$. During the initial part of the $\sigma-\varepsilon$ plot, the process is elastic and we observe a linear relation. For $\varepsilon>\varepsilon_{e}$ (see Fig. 7), the $\sigma-\varepsilon$ relation deviates from linear behavior and we can assume that for $\varepsilon>\varepsilon_{e}$ the strain is provided by the $I / C$ phase transition. For that reason, the $Q_{b}^{-1}$ at $\varepsilon_{e}$ is taken to be the background value. In Fig. 12(a), we show three lines which demonstrate the $\ln \left(Q^{-1}-Q_{b}^{-1}\right)$-ln $\omega$ behavior. Likewise, we obtain the relation $\ln \left(Q^{-1}-Q_{b}^{-1}\right)$-ln $\dot{\varepsilon}$ for fixed $f$ and fixed percentage (46\%) of the $C$ phase; we obtain again three lines in Fig. 12(b). Now we turn our attention to the shear modulus. The initial shear modulus $\mu_{0}$ is defined to be the shear modulus at $\varepsilon=0$, and the relative shear modulus defect is defined to be $\Delta \mu / \mu=\left(\mu_{0}-\mu\right) / \mu_{0}$, which again in this case can be decomposed into two parts, i.e., $(\Delta \mu / \mu)_{\mathrm{SM}}+(\Delta \mu / \mu)_{\mathrm{PI}}$. We plot in Fig. 13 the variation of $(\Delta \mu / \mu) / Q^{-1}$ with $\dot{\varepsilon}$ when the amount of the $C$ phase is $46 \%$. We have a set of such graphs for a series of compositions covering the whole range of phase amount of the $C$ phase. Each curve in Fig. 13 corresponds to a fixed frequency as labeled. Using the data such as shown in Fig. 13, we can make a cross plot at constant $\dot{\varepsilon}$ as shown in Fig. $14,{ }^{2}$ $\ln \left[Q^{-1} /(\Delta \mu / \mu)\right]$ against $\ln \omega$, taking $\dot{\varepsilon}$ as the parameter for each line as marked; note again that there is $46 \% C$ phase in the sample.

From the slopes of lines in Fig. 14, the average value of $l$ is found to be 0.14 (see Table I). We can now employ Eq.

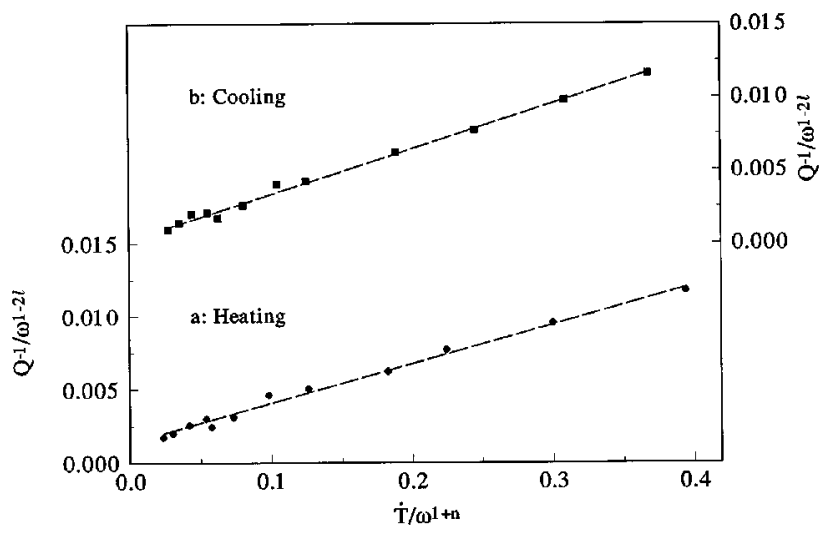

FIG. 11. $Q_{1 / 2}^{-1} /\left(\omega^{1-2 l}\right)$ against $\dot{T}^{n} /\left(\omega^{1+n}\right)$ for the NiTi sample: (a) during heating taking $l=0.15$ and $n=0.35$ and (b) during cooling taking $l=0.12$ and $n=0.30$.
(6) to find the value of $n$. Since there is a one-to-one correspondence between the value of $\varepsilon$ and the phase amount $F$ of the $C$ phase, once $\varepsilon$ is fixed, $F$ is fixed also. We then plot $Q_{F}^{-1} / \omega^{1-2 l}$ against $\dot{\varepsilon}^{n} /\left(\omega^{1+n}\right)$ with a whole set of $Q_{F}^{-1}$ data (with a certain phase fraction $F$ ) and calculate the root-meansquare error $\Delta \delta / \delta$ of the data points about the straight line, which is determined by a simple least-squares fitting. Figure 15 demonstrates two such straight lines corresponding to phase fraction $F=23 \%$ and $46 \%$ as marked. The results of $n$ and $l$ thus deduced pertaining to the smallest values of $\Delta \delta / \delta$ from our analysis for different phase fraction $F$ are listed in Table II covering $F=13.8 \%-55.0 \%$.

\section{DISCUSSION AND CONCLUSIONS}

(1) We have chosen the material NiTi for this investigation because we wish to find out whether the index $n$ is
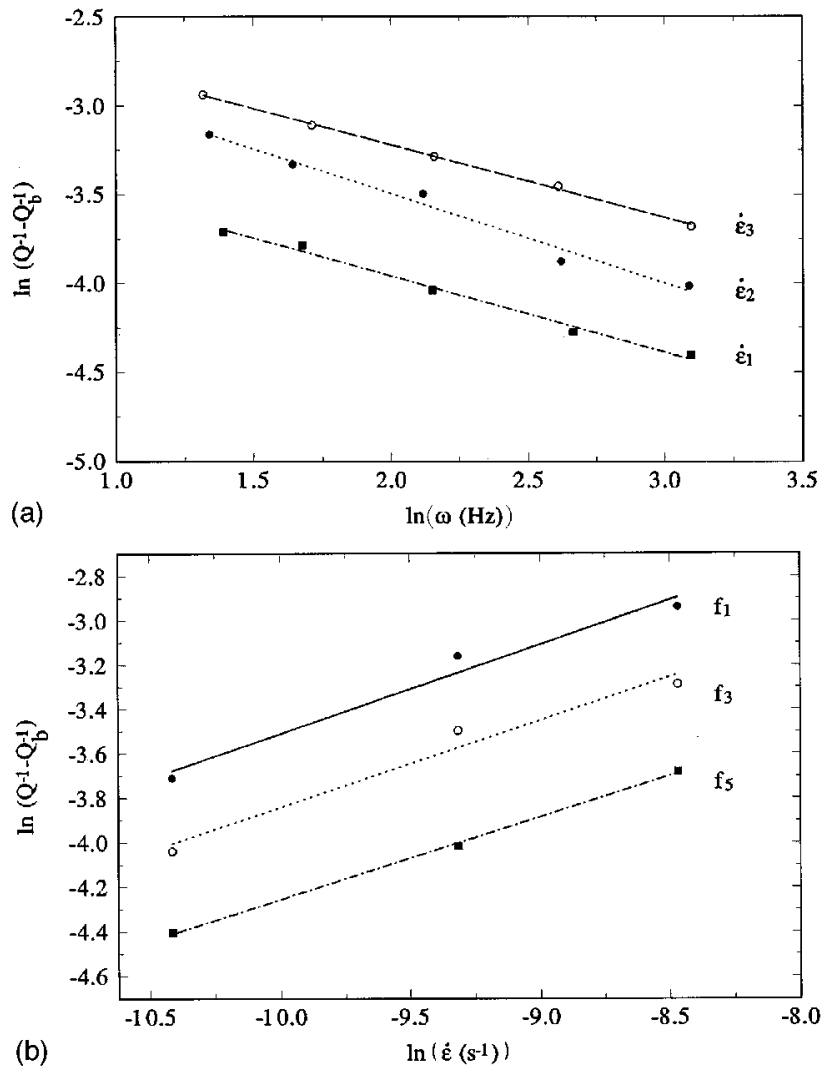

FIG. 12. (a) $\ln \left(Q^{-1}-Q_{b}^{-1}\right)$ vs for $\ln [\omega(\mathrm{Hz})]$ for the $\mathrm{NiFeTi}$ sample: $\quad \dot{\varepsilon}_{1}=3.0 \times 10^{-5} \mathrm{~s}^{-1}, \quad \dot{\varepsilon}_{2}=9.0 \times 10^{-5} \mathrm{~s}^{-1}$, and $\dot{\varepsilon}_{3}=21.3$ $\times 10^{-5} \mathrm{~s}^{-1}$. (b) $\ln \left(Q^{-1}-Q_{b}^{-1}\right)$ against $\ln \dot{\varepsilon}$ for the NiFeTi sample, for $f_{1}=0.6 \mathrm{~Hz}, f_{3}=1.5 \mathrm{~Hz}$, and $f_{5}=3.6 \mathrm{~Hz}$. 


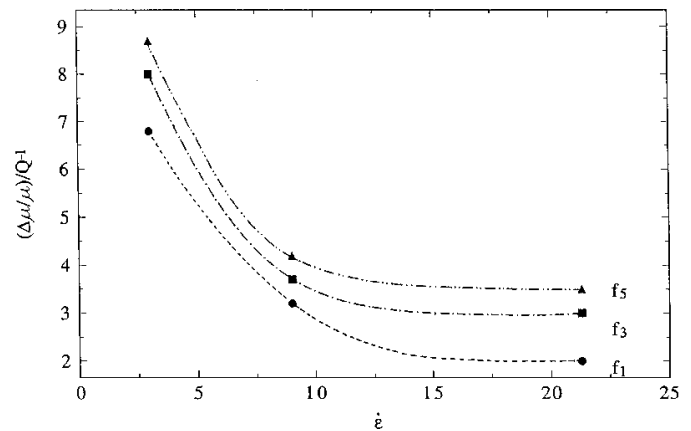

FIG. 13. $(\Delta \mu / \mu) / Q^{-1}-\dot{\varepsilon}$ relation for the NiFeTi sample, for $f_{1}=0.6 \mathrm{~Hz}, f_{3}=1.5 \mathrm{~Hz}$, and $f_{5}=3.6 \mathrm{~Hz}$.

related to the type of phase transition and whether it is related to the lattice coherency on both sides of the PI, taking the FeMn sample ${ }^{3}$ and $\mathrm{VO}_{2}$ sample ${ }^{2}$ as references. The FOPT's of all three samples were caused by temperature variation. It is interesting to note that the FOPT's in both FeMn and NiTi are displacive, ${ }^{29}$ and the values of $n$ found [0.35 (heating, NiTi), 0.33 (heating, FeMn), 0.30 (cooling, NiTi), and 0.47 (cooling, FeMn)] are around 0.30-0.47. It appears that the value of $\Delta T(\sim 50 \mathrm{~K}$ for FeMn and $\sim 5 \mathrm{~K}$ for NiTi) does not affect the value of the net driving index significantly. The FeMn sample has a semicoherent PI, and the NiTi sample has a coherent PI, while $\mathrm{VO}_{2}$ has a noncoherent PI. ${ }^{4,29}$ Since the coupling index $l$ is a measure of the mobility of the PI, we expect the value of $l$ found for NiTi to be smaller than that of $\mathrm{VO}_{2}$, but larger than that of FeMn. This result is indeed observed (see Table I for listing the indices for the three samples). So far, we have deduced two physical indices $l$ and $n$ for three samples only.

(2) Based on energy considerations, we analyze what could have happened in the NiTi sample during a FOPT which has been triggered by temperature variation. Consider a complete heating and cooling cycle of the NiTi sample. Before heating, the lattice has already stored up elastic distortion energy, a characteristic of the martensite phase. When the temperature is raised to drive the $C / I \mathrm{PT}$, the elastic energy released in the course of the PT may enhance the effective driving force, giving a value of $n(0.35)$, which is greater than $n(0.30)$ during the cooling process. During cooling, some energy is stored as elastic distortion energy to complete the cycle.

On the other hand, in a FeMn sample, the martensite

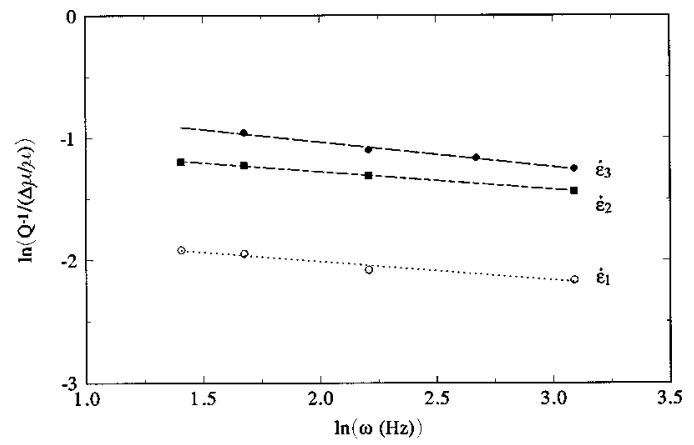

FIG. 14. $\ln \left[Q^{-1} /(\Delta \mu / \mu)\right]$ against $\ln [\omega(\mathrm{Hz})]$ for the $\mathrm{NiFeTi}$ sample: $\quad \dot{\varepsilon}_{1}=3.0 \times 10^{-5} \mathrm{~s}^{-1}, \quad \dot{\varepsilon}_{2}=9.0 \times 10^{-5} \mathrm{~s}^{-1}, \quad$ and $\dot{\varepsilon}_{3}=21.3$ $\times 10^{-5} \mathrm{~s}^{-1}$

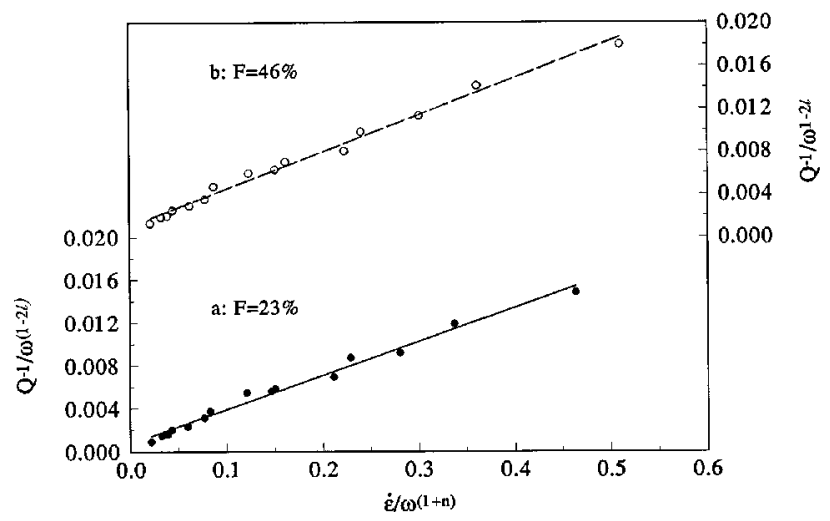

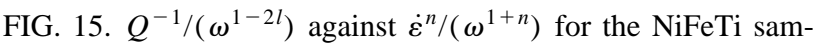
ple: (a) taking $l=0.13, n=0.35$ for $F=23 \%$ and (b) taking $l$ $=0.14, n=0.38$ for $F=46 \%$.

phase and its boundaries contain a significant amount of inelastic distortion. During heating, much of the heat energy is absorbed to eliminate such inelastic distortion. ${ }^{29} \mathrm{We}$ expect that the effective driving force is thus diminished. The result of our combined experiment-theory analysis indicates that indeed the effective driving coefficient $n(0.33)$ is smaller than that during the cooling process $(n=0.47)$. It appears that consequences of our theory are consistent with the experimental findings of the three samples $\mathrm{VO}_{2}, \mathrm{FeMn}$, and NiTi.

(3) The dissipation theory of FOPT's was proposed to describe phase transitions induced by temperature variation in our previous works. ${ }^{1-3}$ Here we have extended the theory to analyze PT's induced by application of stress in Sec. II of this paper. The major difference in the stated two situations arises from the fact that the generalized driving force $\Delta G_{d}$ for the stress-induced thermoelastic MT's is expressible as

$$
\Delta G_{d}=\frac{\varepsilon_{0}}{2}\left(\sigma_{u}-\sigma_{l}\right),
$$

while that in the temperature-induced situation,

$$
\Delta G_{d}=\Delta H\left|T-T_{0}\right| / T_{0} .
$$

In our first application, ${ }^{2,3}$ we chose $T=T_{0}$ at initial time $t^{\prime}$ $=0$ and we can express $\Delta G_{d}=A_{0} \dot{T} t^{\prime}$, where $A_{0}$ is a constant [see Eq. (2) of Ref. 1]. Under such a situation, we can

TABLE II. Indices $n$ and $l$ deduced for different fraction volume.

\begin{tabular}{lcc}
\hline \hline$F(\%)$ & $n$ & $l$ \\
\hline 13.8 & 0.38 & 0.16 \\
18.4 & 0.36 & 0.14 \\
23.0 & 0.35 & 0.13 \\
27.6 & 0.35 & 0.13 \\
32.2 & 0.35 & 0.12 \\
36.8 & 0.36 & 0.13 \\
44.4 & 0.38 & 0.13 \\
46.0 & 0.38 & 0.14 \\
50.6 & 0.38 & 0.15 \\
55.0 & 0.37 & 0.16 \\
\hline \hline
\end{tabular}


measure $Q^{-1}$ and $\mu$ with $\dot{T}=$ const and deduce the driving force of a PI or PT. Here we analyze the dynamics of the stress-induced PI, and $\sigma_{u}-\sigma_{l}$ is not a linear function of $\varepsilon$ in general. As revealed by Fig. 2(a), the $\sigma$ - $\varepsilon$ plot for a typical NiTi sample with almost equal amounts of NiTi demonstrates a shuttle-type $\sigma-\varepsilon$ loop, but when we use a NiTi alloy sample doped with $\mathrm{Fe}$, a "four-straight-sides"-type $\sigma-\varepsilon$ loop results, as shown in Figs. 2(b) and 7. For the NiFeTi sample (see Fig. 7), a certain part of $\left(\sigma_{u}-\sigma_{l}\right)$ is a linear function of $\varepsilon$, implying that we can approximate Eq. (4) by writing $\Delta G_{d}=\left(\varepsilon_{0} / 2\right)\left(\sigma_{u}-\sigma_{l}\right) \approx A_{0} \varepsilon=A_{0} \dot{\varepsilon} t^{\prime}$. As in the temperature-variation situation, the last equality holds when we have a correct choice of the zero point of time while keeping $\dot{\varepsilon}=$ const. This expression is identical in form to $\Delta G_{d}=A_{0} \dot{T} t^{\prime}$, with $\dot{\varepsilon}$ replacing $\dot{T}$. Thus Eqs. (4) and (5) are valid under the approximation that $\left(\sigma_{u}-\sigma_{l}\right)$ is a linear function of $\varepsilon$, to the lowest order of approximation.

(4) Based on the analysis presented in Sec. II, we have found that while the $\sigma-\varepsilon$ plot for the $\mathrm{Ni}_{49} \mathrm{Ti}_{51}$ sample is not a straight line, the $\sigma_{l^{-}} \varepsilon$ and $\sigma_{n^{-}} \varepsilon$ plots for the NiFeTi sample are straight lines in a certain region. In such a region $\varepsilon$ is a linear function of time based on Eq. (4): $\Delta G_{d}=\left(\varepsilon_{0} / 2\right)$ $\times\left(\sigma_{u}-\sigma_{l}\right)$. For this particular reason, we have chosen the NiFeTi sample to test our theory developed in Sec. II. In fact, we observe from Figs. 1(b) and 3 that the $I / C$ and $C / M$ phases are separated clearly in the strain domain and we can analyze $I / C$ as a single FOPT. Since only $1 \%$ of $\mathrm{Fe}$ is doped to the NiTi specimen with around equal amounts of $\mathrm{Ni}$ and $\mathrm{Ti}$, we hope thereby to test the theory along two parallel lines (temperature variation and stress application).

(5) The values of the coupling index $l$ and the effective driving index $n$ for the above two specimens are found to be very similar $[l=0.12-0.15(\mathrm{NiTi})$ and $0.14(\mathrm{NiFeTi}) ; n$ $=0.3-0.35(\mathrm{NiTi})$ and $0.36(\mathrm{NiFeTi})]$. Such a result indicates that $l$ and $n$, which represent certain characteristics of the PI, remain practically the same, irrespective of the methodology of initiation of the PT (i.e., temperature variation or stress induced). This conclusion appears to be consistent with intuition. We believe the indices $n$ and $l$ are indeed fingerprints of FOPT in solids.

\section{ACKNOWLEDGMENTS}

The work of J.X.Z. and Z.C.L. was supported by the Chinese National Science Foundation and the Guangdong Province Natural Science Foundation. P.C.W.F. is Visiting Professor at Zhongshan University, and is partly supported by a CRCG grant and RGC grants in Hong Kong for this project.
${ }^{1}$ J. X. Zhang, P. C. W. Fung, and W. G. Zeng, Phys. Rev. B 52, 268 (1995).

${ }^{2}$ J. X. Zhang, Z. H. Yang, and P. C. W. Fung, Phys. Rev. B 52, 278 (1995).

${ }^{3}$ P. C. W. Fung, J. X. Zhang, Y. Lin, K. F. Liang, and Z. C. Lin, Phys. Rev. B 54, 7074 (1996).

${ }^{4}$ N. R. Rao and K. J. Rao, Phase Transitions in Solids (McGrawHill, New York, 1978).

${ }^{5}$ W. J. Buehler, J. V. Gilfrich, and R. C. Wiley, J. Appl. Phys. 34, 1475 (1963).

${ }^{6}$ F. E. Wang, W. J. Buehler, and S. J. Pickart, J. Appl. Phys. 36, 3232 (1963).

${ }^{7}$ C. M. Hwang et al., Res. Mech. 10, 1 (1984).

${ }^{8}$ K. Otsuka and K. Shimizu, Int. Metall. Rev. 31, 93 (1986).

${ }^{9}$ T. Saburi, M. Yoshida, and S. Nenno, Scr. Metall. 18, 363 (1984).

${ }^{10}$ J. X. Zhang and J. H. Li, Acta Phys. Sin. 37, 363 (1988).

${ }^{11}$ Z. Y. Xu, Martensitic Transformation and Martensite (Academic, Beijing, 1980), Chap. 8.

${ }^{12}$ R. V. Krisbnan, L. Delaey, H. Tas, and H. Warlimont, J. Mater. Sci. 9, 1536 (1994).

${ }^{13}$ C. W. Hwang, M. Meicjle, M. Salamon, and C. M. Wayman, Philos. Mag. A 47, 9 (1983).

${ }^{14}$ J. X. Zhang, G. M. Lin, Y. S. Huang, X. Lin, Q. H. Jiang, H. B. Yang, Z. H. Shao, and W. Q. Hong, Acta Zhongshan University 24(2), 1 (1985).

${ }^{15}$ J. H. Li and J. X. Zhang, J. Phys. (France) Colloq. 46, C10-477 (1985).
${ }^{16}$ J. X. Zhang and X. J. Li, Acta Phys. Sin. 36, 847 (1987).

${ }^{17}$ J. Stoiber, J.-E. Bidaux, and R. Gotthardt, Adv. Mater. Opt. Electron. 42, 4059 (1994).

${ }^{18}$ R. J. Wasilewski et al., Scr. Metall. 5, 127 (1971).

${ }^{19}$ K. Otsuka, T. Sawamura, and K. Shimizu, Phys. Status Solidi A 5, 457 (1975).

${ }^{20}$ G. D. Sandrock, A. J. Perkins, and R. F. Hehemann, Metall. Trans. A 2, 2769 (1971).

${ }^{21}$ H. C. Ling and R. Kaplow, Metall. Trans. A 12, 2101 (1981).

${ }^{22}$ L. Z. Luo and J. X. Zhang, in Proceedings of the International Conference on SMA-86, edited by Y. Y. Chu (Academic, Beijing, 1986), p. 115.

${ }^{23}$ G. M. Lin and D. Y. Li, Acta Zhongshan University 24(4), 45 (1985).

${ }^{24}$ L. Z. Luo and J. X. Zhang, J. Phys. (France) Colloq. 46, C10-649 (1985).

${ }^{25}$ J. X. Zhang and L. Z. Luo, Acta Phys. Sin. 37, 353 (1988).

${ }^{26}$ H. Funakubo, Shape Memory Alloy (Industrial Books, New York, 1984), p. 83.

${ }^{27}$ M. Nishida and T. Honma, Scr. Metall. 18, 1293 (1984).

${ }^{28}$ K. F. Liang, Z. C. Lin, and J. X. Zhang, in Proceedings of ICIFUAS-9, edited by T. S. Kê (Academic, Beijing, 1989), p. 365.

${ }^{29}$ Z. Y. Xu, Martensitic Transformation and Martensite (Academic, Beijing, 1980), Chaps. 1 and 8. 